\title{
Asthma and chronic obstructive pulmonary disease overlap syndrome: An update
}

\author{
Ming Lu, Wan-zhen Yao \\ Department of Respiratory Medicine, Peking University Third Hospital, Beijing 100191, China
}

The scientific committees of Global Initiative for Chronic Obstructive Pulmonary Disease (GOLD) and Global Initiative for Asthma (GINA) released their updated guidelines, respectively, in January and May of 2014, referred to as GOLD 2014 and GINA 2014..$^{[1,2]}$

After convening a panel discussion, the GINA and GOLD scientific committees jointly proposed a term "asthma-COPD overlap syndrome" (ACOS) based on current publications and expert consensus, which received widespread attention as a backgrounder in GOLD 2014, and later as a detailed full text in GINA 2014.

Although asthma and chronic obstructive pulmonary disease (COPD) are two of the most common airway obstructive diseases in clinics, they differ from each other in clinical characteristics and pathogenesis. Asthma is a heterogeneous disease more likely to affect children and teenagers, often accompanied by allergic rhinitis, urticaria, and other allergic diseases, and associated with a family history of atopy. Clinically, asthma is manifested as volatile severity of wheezing over time. Asthmatic patients usually have airway inflammation predominated by eosinophils and $\mathrm{CD}^{+}$ $\mathrm{T}$ lymphocyte infiltration; variable and reversible airflow limitation; and, between two episodes of asthma attack, basically normal lung functions. The patients often respond well to corticosteroids. On the other hand, COPD is associated with aberrant chronic inflammation of the airway and lungs arising from exposure to noxious particles and gases, and is more likely to affect the population aged above 40 . COPD patients have dyspnea on exertion, airway inflammation predominated by infiltration of neutrophils, CD8+ T lymphocytes and macrophages, and persistent but progressive airflow limitation as shown by lung function test (LFT). They usually respond poorly to corticosteroids, and the outcomes are often disappointing. Despite a great deal of differences between asthma and COPD, both share many similarities. Patients with either one of these entities may present with clinical symptoms such as chronic cough, sputum, and exertional dyspnea. LFTs may reveal obstructive ventilatory dysfunction for both. It has been demonstrated that the airflow limitation was not fully reversible in $16 \%$ of asthma patients over a 20-year follow-up, ${ }^{[3]}$ and that positive bronchial provocation test (BPT) and airway hyperresponsiveness (AHR) may also be indicated in a considerable percentage of COPD patients. ${ }^{[4]}$ Pulmonary diffusion capacity was once considered as a physiological measure that would best distinguish between asthma and COPD, but unfortunately, a level of carbon monoxide diffusing capacity (DLCO) less than 80\% predicted value only yielded $77 \%$ sensitivity and $71 \%$ specificity. ${ }^{[5]}$ In addition, Jatakanon and colleagues ${ }^{[6]}$ found neutrophils may be the predominating cells in airway secretions from a number of patients with mild to severe asthma, ${ }^{[7]}$ whereas sputum eosinophilic counts may also increase in acute exacerbation of COPD. Pathologically, both asthma and COPD may exhibit airway remodeling, which involves subepithelial fibrosis, epithelial goblet cell metaplasia, and hyperplasia or hypertrophy of smooth muscle cells.

Based on these understandings, identifying asthma versus COPD should not be difficult for typical cases, but this is not always easy for a certain subset of patients. 
The probable copresence of asthma and COPD in an individual may further complicate this dilemma. It is not unusual that a patient may present concomitantly with clinical features of asthma and COPD. The prevalence of ACOS, reported between $15 \%$ and $55 \%$ in clinical studies on asthma or COPD, ${ }^{[8,9]}$ has raised concerns among clinicians for a long time. Readers might still remember the 3-circle Venn diagram defining asthma, chronic bronchitis, emphysema, COPD, and airflow obstruction in American Thoracic Society (ATS) COPD guidelines first released in 1995. ${ }^{[10]}$ Intersection of the 3 circles produced a total of 11 subsets (syndromes), including 6 with an overlap nature. In 2007 China COPD guidelines, it was clearly stated that asthma and COPD may overlap and coexist in certain patients. ${ }^{[1]]}$ The Dutch hypothesis maintains that all airflow obstructive diseases (including asthma and COPD) are different expressions of a single disease that originates from a common background of genetic susceptibility to allergy and the AHR, ${ }^{[12]}$ and in response to the changes in environmental influences coupled with many other factors like smoking, allergen exposure, and recurrent airway infections, evolves into various individual or overlapping phenotypes. Therefore, according to the Dutch hypothesis, ACOS may be a novel phenotype of nonspecific chronic airflow obstructive disease independent of asthma and COPD. However, the Dutch hypothesis has never been widely accepted in the community of respiratory medicine, where most specialists currently hold that asthma and COPD are two different disease entities, and it is no surprise that both may concomitantly appear in a patient since they are common disorders. Thus, the "overlap" indicated by the GINA 2014 term "asthma and COPD overlap syndrome" may be implicating more about the coexistence of these two disease entities, so as to go in line with clinical conceptions.

Nearly all clinical trials excluded patients with concomitant COPD and asthma. Accordingly, the current evidencebased information about ACOS is lacking. In fact, the GINA 2004 definition for ACOS is not a formal one, ${ }^{[1,2]}$ but rather, a description of clinical features based on individual definitions of asthma and COPD. These clinical features of ACOS, in essence, reflect part of asthma and part of COPD, or alternatively, the coexistence of asthma and COPD in the same patient. While the imaging findings in ACOS patients may show prominent signs of expiratory air trapping, ${ }^{[13]}$ some studies also showed more airway wall thickening than emphysema. ${ }^{[14]}$ ACOS patients have airway inflammation similar to that in COPD patients, as featured by increased sputum neutrophils; but meanwhile, the sputum eosinophil counts are also elevated in ACOS compared with COPD, although lower than those in asthma alone. ${ }^{[15]}$
A well-recognized set of diagnostic criteria for ACOS is yet to develop. GINA 2014 offers no definite criteria for the diagnosis more than recommendation on considering ACOS in patients with certain clinical scenarios. In general, it recommends collecting clinical features that favor diagnosis of asthma or COPD as the first step, and then comparing a total of 11 feature items supportive respectively of asthma and COPD diagnoses. When the number of supportive feature items seems comparable between asthma and COPD, a diagnosis of ACOS should be considered. Following this, a ratio of postbronchodilator forced expiratory volume in one second (FEV1) over forced vital capacity (FVC) less than 0.7, plus reversible airflow limitation (post-bronchodilator FEV1 increase $\geq 12 \%$ and $\geq 200 \mathrm{~mL}$ ) or significantly reversible airflow limitation (post-bronchodilator FEV1 $\geq 15 \%$ and $\geq 400 \mathrm{~mL}$ ), may confirm the diagnosis of ACOS. A consensus document from Spain stated that, for COPD patients, a diagnosis of ACOS may be determined if at least two of the major criteria and two of the minor criteria can be fulfilled, as follows. ${ }^{[16]}$ The major criteria include: (1) very positive bronchodilator test (increase in postbronchodilator FEV1 $\geq 15 \%$ and $\geq 400 \mathrm{~mL}$ from baseline); (2) eosinophilia in sputum; and (3) personal history of asthma before the age of 40 . The minor criteria include: (1) elevated total IgE; (2) personal history of atopy; and (3) positive bronchodilator test on two or more occasions (increase in post-bronchodilator FEV1 $\geq 12 \%$ and $\geq 200$ $\mathrm{mL}$ from baseline). Although more epidemiological data are needed to validate the generality of these criteria for other parts of the world, according to the above criteria, a diagnosis of ACOS in the first place should fulfill a postbronchodilator FEV1/FVC $<0.7$, that is, as required for the diagnosis of COPD. In addition to the LFT findings, a considerable body of clinical features for asthma has to be present for diagnosing ACOS. In this regard, the GINA 2014 and the Spain consensus document on COPD are believably more inclined to the opinion that ACOS is the coexistence of asthma and COPD.

Clinically, ACOS is commonly presented in several types: (1) history of childhood asthma and smoking, gradual development of fixed airflow obstruction (postbronchodilator FEV1/FVC < 0.7), accompanied by reversible airflow limitation after the age of 40 . These patients also present with features of childhood-onset asthma and smoking-induced COPD appearing during the middle to advanced age, and thus may be considered as having asthma-COPD overlap; $;^{[8,15]}$ (2) history of smoking and exposure to harmful substances, progressive deterioration of exertional dyspnea after the age of 40 , accompanied by recurrent episodes of wheezing following exposure to allergenic factors with good response to glucocorticoids, and fixed airflow obstruction 
with reversible airflow limitation on LFT. Although these patients show no signs of childhood atopy, their presentations may be consistent with late-onset asthma, a phenotype recognized by many investigators in recent years, which is typically associated with a negative history of allergies in childhood and among family members. ${ }^{[17]}$ In this regard, the patients may as well be considered as concomitantly having COPD and late-onset asthma; (3) a history of childhood asthma, no smoking habits or no exposure to hazardous substances, good or bad asthma control, and development of persistently fixed airflow obstruction after the age of 40 . These patients do not have features of COPD induced by cigarette smoking or other factors. Although they may finally develop persistent airflow limitation, in clinical practice, this is usually considered to be resulting from airway remodeling caused by chronic asthma, and hence asthma is ultimately the diagnosis. Unless these patients have emphysema, bullae, or significantly reduced DLCO on LFT, this scenario does not represent an overlap of asthma and COPD.

So far, large clinical trials of drugs on asthma or COPD have not included patients "without clearly identified asthma or COPD," which adds to the lack of evidence-based data for treatment of ACOS. Accordingly, clinical practice with ACOS could only refer to current guidelines for asthma and COPD management, so as to achieve a comprehensive adoption of various treatment recommendations. ${ }^{[2,16]}$ Given the important role of inhaled corticosteroids (ICS) in the treatment of asthma, and of long-acting bronchodilators in COPD, early use of combination therapy with ICS and long-acting bronchodilators should be recommended to relieve symptoms, improve lung function, and reduce acute exacerbation. In severe cases, it is recommended to use three agents in the combination therapy, that is, ICS + long-acting inhaled $\beta$-adrenergic receptor agonist + inhaled long-acting anticholinergic drugs. Monotherapy with longacting $\beta 2$-agonists alone should be avoided.

In summary, the term ACOS jointly proposed by GOLD 2014 and GINA 2014 may have clinical implications. For patients with clinical features of both asthma and COPD, these expert consensuses at the least offer acceptable taxonomy of the disorder. In the future, more epidemiological studies, basic research, and multicenter clinical trials are warranted to provide evidence for the underlying mechanisms, definition, and standardized diagnosis and management of ACOS.

\section{REFERENCES}

1. Global Strategy for the diagnosis, management, and prevention of chronic obstructive pulmonary disease [EB/OL]. http: //www. ginasthma. org/ local/uploads/files/ GINA_Report_2014_Aug12. pdf. Accessed on December 8, 2014.

2. Global Initiative for Chronic Obstructive Lung Disease (GOLD). Global Strategy for the Diagnosis, Management and Prevention of COPD [EB/ OL]. http: //www. goldcopd. org/uploads/users/files/GOLD_Report_2015_Feb18. pdf. Access on August 2, 2015.

3. Vonk JM, Jongepier H, Panhuysen C, Schouten J, Bleecker E, Postma D. Risk factors associated with the presence of irreversible airflow limitation and reduced transfer coefficient in patients with asthma after 26 years of follow up. Thorax 2003; 58: 322-7.

4. Calverley PM, Burge PS, Spencer S, Anderson JA, Jones PW. Bronchodilator reversibility testing in chronic obstructive pulmonary disease. Thorax 2003; 58: 659-64.

5. Magnussen H, Richter K, Taube C. Are chronic obstructive pulmonary disease (COPD)and asthma different diseases? Clin Exp Allergy 1998; 28: 187-94.

6. Jatakanon A, Uasuf C, Maziak W, Lim S, Chung KF, Barnes PJ. Neutrophilic inflammation in severe persistent asthma. Am J Respir Crit Care Med 1999; 160(5 Pt 1): 1532-9.

7. Zhu J, Qiu YS, Majumdar S, Gamble E, Matin D, Turato G, et al. Exacerbations of Bronchitis: bronchial eosinophilia and gene expression for interleukin-4, interleukin-5, and eosinophil chemoattractants. Am J Respir Crit Care Med 2001;164: 109-16.

8. Zeki AA, Schivo M, Chan A, Albertson TE, Louie S. The Asthma-COPD overlap syndrome: a common clinical problem in the elderly. J Allergy 2011; 2011: 861926.

9. Marsh SE, Travers J, Weatherall M, Williams MV, Aldington S, Shirtcliffe PM, et al. Proportional classifications of COPD phenotypes. Thorax 2008; 63: 761-7.

10. American Thoracic Society. Standards for the diagnosis and care of patients with chronic obstructive pulmonary disease. Am J Respir Crit Care Med 1995; 152(5 Pt 2): S77-121.

11. The guideline for the diagnosis and treatment of chronic obstructive pulmonary disease. Chin J Tuberc Respir Dis 2007; 1: 8-17.

12. Orie NG. The dutch hypothesis. Chest 2000; 117(5 Suppl 1): $299 \mathrm{~S}$.

13. Hardin M, Silverman EK, Barr RG, Hansel NN, Schroeder JD, Make BJ, et al. The clinical features of the overlap between COPD and asthma. Respir Res 2011; 12: 127.

14. Hardin M, Cho M, McDonald ML, Beaty T, Ramsdell J, Bhatt S, et al. The clinical and genetic features of COPD-asthma overlap syndrome. Eur Respir J 2014; 44: 341-50.

15. Gibson PG, Simpson JL. The overlap syndrome of asthma and COPD: what are its features and how important is it? Thorax 2009; 64: 728-35.

16. Soler-Cataluña JJ, Cosío B, Izquierdo JL, López-Campos JL, Marín JM, Agüero R, et al. Consensus document on the overlap phenotype COPDasthma in COPD. Arch Bronconeumol 2012; 48: 331-7.

17. Ariano R, Panzani RC. Late onset asthma in the elderly and its relationship with atopy. Eur Ann Allergy Clin Immunol 2012;44: 35-41.

How to cite this article: Lu M, Yao W. Asthma and chronic obstructive pulmonary disease overlap syndrome: An update. J Transl Intern Med 2015; 3: 144-146.

\section{Conflicts of Interest}

None declared. 